\title{
5 Länderkontext - Traditionelle Landrechte und das chieftaincy-System in Ghana
}

Ziel dieses Kapitels ist es, einen Überblick über die historische Entwicklung der Landrechte und die Bedeutung traditioneller Autoritäten in Ghana zu geben. Besonderes Augenmerk liegt auf der Darstellung traditioneller Landverwaltungssysteme, dessen Verständnis grundlegend für die spätere Analyse ist.

\subsection{Landrechte und die koloniale Erfindung von Traditionen}

Seit Beginn der Kolonialisierung strebte die Britische Krone danach, Zugang und Nutzung von Land zu kontrollieren, um über die Vergabe von Bergbaukonzessionen und Landverkäufen an der florierenden Goldförderung und den Einnahmen aus der Kakaoproduktion teilhaben zu können (Amanor 2005). Geschäfte mit landwirtschaftlichen Nutzflächen nahmen in der heutigen Volta Region im Südosten Ghanas bereits in den frühen Jahren des 19. Jahrhunderts zu, als Palmöl zu einem gefragten Rohmaterial für die industrielle Weiterverarbeitung wurde. Diese Entwicklung verstärkte sich in den 1890er Jahren, in denen Kakao Palmöl als wichtigste Exportpflanze ersetzte. Die 1890er Jahre waren zudem eine Zeit intensiver Goldspekulation, in der sich mehr als 400 Bergbauunternehmen an der Goldküste niederließen und große Waldflächen aneigneten (Amanor 1999: 46). Die fortschreitende Kommodifizierung von Land missfiel der Kolonialverwaltung, da sie selbst nicht daran beteiligt war und kaum eine Möglichkeit hatte, die Ausweitung der kapitalistischen Produktion zu regulieren und Regierungseinnahmen daraus zu generieren. 1884 versuchte die Kolonialregierung daher den Crown Lands Bill einzubringen. Dieses Gesetz sollte »ungenutztes« Land (so genanntes waste land) - unbewirtschaftetes Land, auf das niemand einen formalisierten Rechtsanspruch 
hatte -, sowie Forstflächen und Mineralien unter die Kontrolle der Britischen Krone bringen. Diese sollte fortan das Recht haben, auf unbewirtschaftetem Land Bergbaukonzessionen an internationale Unternehmen zu erteilen. Traditionelle Landnutzer*innen sollten das Land weiterhin bestellen dürfen, ohne jedoch die Möglichkeit zu haben, Landrechte an Fremde - insbesondere europäische Investoren - zu vergeben (ebd.). Der Erlass des Crown Lands Bill scheiterte jedoch am Widerstand eines Zusammenschlusses aufstrebender Intellektueller. Sie argumentierten, jedes Stück Land habe eine*n Besitzer"in. In Folge des anhaltenden Widerstands annullierte die Kolonialregierung den Crown Lands Bill und ersetzte ihn 1897 durch den Lands Bill. Dieser unterschied sich im Wesentlichen dadurch, dass die Kolonialregierung den umstrittenen Begriff waste land ausließ und stattdessen Anspruch auf die Kontrolle über öffentliches Land (public land) erhob. Der Lands Bill gestand den chiefs weiterhin Rechte an Land $\mathrm{zu}$, die ihnen ermöglichten Land an andere Afrikaner*innen, nicht jedoch an Europäer*innen zu vergeben. Afrikaner*innen konnten generell nur temporäre Rechte (settler rights) erlangen. Vererbbare Eigentumsrechte, die auf britischem Recht beruhten, konnte allein die Kolonialregierung vergeben. Auch gegen den Lands Bill gab es erheblichen Widerstand, insbesondere seitens der chiefs und der aufstrebenden intellektuellen Handelsklasse der Goldküste. Sie gründeten gemeinsam die Aborigines' Rights Protection Society, deren Ziel es war, die Interessen der chiefs sowie der kolonialisierten Bevölkerung der Goldküste zu vertreten (Amanor 2005: 103). Die Alternative zum gescheiterten Lands Bill stellte für die Kolonialregierung ein System der Native Administration dar, das sie 1927 mit der Native Administration Ordinance verkündeten. Dieses System baute auf den Prinzipien der für die britische Kolonialmacht typischen indirekten Herrschaft (indirect rule) auf (Crowder 1964). Im Rahmen dieser übte die Kolonialregierung Macht über bereits bestehende örtliche und traditionelle Herrschaftsstrukturen aus. Beruhend auf dem System der indirekten Herrschaft schuf die britische Kolonialregierung in Ghana ein Landnutzungssystem, das Gewohnheitsrecht aufrechterhielt, gleichzeitig aber neue formal-staatliche Gesetze nach englischem Recht einführte (Aryeetey 2007: 11). Das ermöglichte der Kolonialregierung, traditionelle Autoritäten - die bisher in den Widerstand eingebunden waren - als wichtige Verbündete $\mathrm{zu}$ gewinnen und in ihr Herrschaftssystem $\mathrm{zu}$ integrieren. Für die Aufgabe der Landverwaltung in den südlichen Regionen wählte die Kolonialregierung unter den bestehenden traditionellen Autoritäten einer Gegend eine Person aus, die sie zum paramount chief ernannte. In den nördlichen Regionen unterschied sich das Vorgehen, da dort bislang kein chieftaincy-System 
wie im Süden bestand, sondern spirituelle Führer (earth priests) das Land treuhänderisch für lokale Gemeinschaften verwalteten (Lund 2008). Diese ignorierte die Kolonialregierung in ihrem Bestreben, Kontrolle über Land zu erlangen und ernannte stattdessen einflussreiche Kaufleute sowie andere reiche und mächtige Personen zu paramount chiefs (Kasanga/Kotey 2001: 13f.; ObengOdoom 2016: 666). Paramount chiefs bestimmten fortan - kontrolliert durch die Kolonialmacht - die Ausgestaltung von Landverhältnissen, indem sie Verordnungen erließen und festlegten, welche Regeln und Normen in Bezug auf die Landnutzung als gewohnheitsrechtlich anzusehen waren (Boone 2015: 174). Dabei handelte es sich im Wesentlichen um die »Erfindung von Traditionen« (Ranger 1983), auf die sich Kolonialregierung und traditionelle Autoritäten verständigten (Amanor 2008: 63f.). Dies war problematisch, da der chief nicht zwangsläufig die gleiche Vorstellung traditioneller Rechte wie andere Mitglieder der Gemeinschaft hatte (Obeng-Odoom 2016: 665). In einigen Fällen machten chiefs gegenüber der Kolonialregierung auch vorsätzlich falsche Angaben zum Verlauf von Grenzen, um ihre territoriale Macht auszuweiten (Lentz 2010: 74). Kooperationsunwillige oder widerständige chiefs enthob die Kolonialmacht (teilweise gewaltsam) ihres Amts. Anderen, die sich ihr verbunden zeigten, verhalf sie durch Unterstützung in der Beseitigung von Rivalen zur Ausweitung ihrer (territorialen) Macht (von Trotha 1996: 80). Die indirekte Herrschaft über traditionelle Autoritäten ermöglichte es der Britischen Krone, Kontrolle über Land zu erlangen (Ubink 2008a: 22). Gleichzeitig führte dieses System zu einer gestärkten Position traditioneller Autoritäten in der Landvergabe, die über die Kolonialzeit hinaus Bestand hatte. Narrative, die das ideologische Konstrukt aufrechterhalten, dass traditionelle Autoritäten - und nicht die Landnutzer*innen - die Eigentümer*innen des Lands seien, legitimieren bis heute die Landvergabe durch traditionelle Autoritäten. Diese Narrative beschreiben jedoch keine prä-koloniale Realität, sondern reproduzieren koloniale Praxis (Berry 2013: 37).

\subsection{Traditionelle Herrschaft und Landrechte im postkolonialen Ghana}

Mit dem Ende der Kolonialzeit und in den ersten Jahren der Unabhängigkeit änderte sich das ghanaische Landrecht zunächst nicht. Obwohl die unterschiedlichen Regierungsparteien sich in ihrer Ausrichtung deutlich unterschieden, strebten sie keine Veränderung der Landpolitik an. Einzig der So- 
zialist Kwame Nkrumah, Ghanas erster Präsident nach der Unabhängigkeit, bildet hier eine Ausnahme. Er forderte eine Befreiung von traditionellem Elitismus (Nkrumah 1961) und versuchte die wirtschaftliche Macht der chiefs zu brechen, indem er ihnen das formelle Recht absprach, Land zu verwalten und Ansprüche auf Pacht oder anderen Einnahmen zu erheben (Ubink 2008c: 10). Mit dem Administration of Lands Act (Act 123) von 1962 übertrug er die Landverwaltung staatlichen Institutionen. Strukturell änderte sich dadurch jedoch nichts und das System der Landverwaltung blieb in seiner vorherigen Form mehr oder weniger bestehen. Denn obwohl der Staat die Verwaltung von Land übernahm, kamen die Einnahmen daraus letztendlich weiterhin den chiefs zugute (Obeng-Odoom 2016: 667). Nkrumahs kritische Haltung gegenüber traditioneller Herrschaft zog somit de facto keine Einschränkung der Macht der chiefs in der Landverwaltung nach sich. Bis auf kleinere Gesetzesänderungen veränderte sich das Landrecht nach dem Ende der Kolonialzeit bis in die 1980er Jahre kaum (ebd.).

\section{Landreformen und die erneute Stärkung traditioneller Herrschaft}

Erst seit den späten 1980er Jahren unter Präsident Jerry John Rawlings erlebte das Landverwaltungssystem größere Veränderungen. Diese waren Teil einer Welle von Landreformen, die insbesondere internationale Geber- und Finanzinstitutionen wie die Weltbank auf dem afrikanischen Kontinent förderten. Befürworter*innen sahen diese Reformen als wichtigen Schritt für die Sicherung von Landrechten und dadurch einen besseren Zugang zu Krediten, höhere Bildungschancen und die Verringerung von Armut (World Bank 2004). Kritiker"innen hingegen argumentierten, die Reformen kämen hauptsächlich internationalen Unternehmen zugute, die Land auf Kosten der lokalen Bevölkerung aneigneten, um landwirtschaftliche Nutzflächen in Investitionsflächen umzuwandeln (Elhadary/Obeng-Odoom 2012: 59).

Zeitgleich mit der Ausarbeitung von Landreformen gewannen traditionelle Autoritäten - denen seit dem Ende der Kolonialzeit wenig Aufmerksamkeit zuteilwurde - wieder an Einfluss (Buur/Kyed 2007; Englebert 2002b). Trotz staatlicher Bestrebungen, traditionelle Herrschaft zu schwächen, wies diese über die Jahre eine beachtliche Resilienz auf (Ubink 2008c). Traditionellen Autoritäten gelang es sukzessive mehr Einfluss über informelle bottom-up-Prozesse zu erlangen, indem sie sich in Organisationen engagierten oder in größeren ökonomischen Netzwerken zusammenschlossen. Auf diese Weise etablierten sie sich mit der Zeit als erfolgreiche Geschäftsleute mit gu- 
ten nationalen und internationalen Beziehungen. Wirtschaftlicher Erfolg und Netzwerke ermöglichten es ihnen, verstärkt Einfluss in der lokalen oder nationalen Politik auszuüben. Darüber hinaus konsolidierten traditionelle Autoritäten ihre Macht, indem sie auf lokalpolitischer Ebene informell staatliche Aufgaben übernahmen - sowohl anstelle von als auch in Ergänzung zu den Behörden. In Kontexten, in denen staatliche Institutionen schwach oder nicht vorhanden waren, ersetzten traditionelle Autoritäten sie und erwiesen sich darüber als wichtige Dienstleister*innen für die lokale Bevölkerung (Kyed/Buur 2007: 2).

Im Zuge des Paradigmenwechsels internationaler Geberorganisationen hin zu lokalen und dezentralen Verwaltungsstrukturen gewannen traditionelle Autoritäten als Schlüsselakteure wieder zunehmend an politischer Relevanz (Kirst 2019). Die Regierung Ghanas erkannte diese Relevanz an und räumte traditioneller Herrschaft mit der Verfassung von 1992 einen formellen Status ein. Traditionelle und staatliche Herrschaft ist dabei strikt getrennt. Diese Trennung wird unter anderem dadurch deutlich, dass dem Parlament untersagt ist, sich in chieftaincy-Fragen einzumischen. Das Parlament kann keine chiefs ernennen, ihre Anerkennung verweigern oder Gesetze erlassen, die der chieftaincy-Institution schaden (Republic of Ghana 1992: Art. 270). Darüber hinaus garantiert die Verfassung den Fortbestand des National House of Chiefs. In dieses werden jeweils fünf traditionelle Autoritäten aus den zehn Regional Houses of chiefs abgeordnet, um die Interessen der unterschiedlichen traditionellen Gemeinschaften gegenüber dem Staat sowie innerhalb des chieftaincy-Systems zu vertreten. Sie sollen staatliche Akteure zu chieftaincy-Fragen beraten, traditionelles Recht kodifizieren, zur Harmonisierung von staatlichem und traditionellem Recht beitragen und diesbezüglich mögliche Konflikte identifizieren. Des Weiteren sollen sie eine Beurteilung aktueller traditioneller Gepflogenheiten und Bräuche gewährleisten und darauf hinarbeiten, dass sozial nachteilige gewohnheitsrechtliche Normen und Regeln nicht fortbestehen (ebd.: Art. 271, Art. 272). Abgesehen von der beratenden Funktion traditioneller Autoritäten untersagt die Verfassung ihnen, sich aktiv in der Parteipolitik zu engagieren. Traditionellen Autoritäten ist es nicht erlaubt politische Ämter zu bekleiden. Streben sie einen politischen Posten an, müssen sie ihre Position als chief aufgeben (ebd.: Art. 276). Diese Maßnahme soll die politische Einflussnahme auf traditionelle Herrschaft verhindern (Elhadary/Obeng-Odoom 2012).

In Bezug auf die Verwaltung von Land erkennt die Verfassung die $\mathrm{Zu}$ ständigkeit traditioneller Autoritäten an (Republic of Ghana 1992: Art. 267, 
Abs. 1) und schreibt ihnen die Rolle von Treuhändern der jeweiligen Gemeinschaft zu, die sich verpflichten zum Wohl und Nutzen ihrer Gemeinschaft zu handeln (ebd.: Art. 36, Abs. 8). Die Verteilung der Einnahmen aus dem Land überträgt sie dem staatlichen Office of the Administrator of Stool Lands (OASL) (ebd.: Art. 267, Abs. 2). Laut verfassungsrechtlich festgelegtem Verteilungsschema behält dieses zehn Prozent aller Einnahmen zur Deckung administrativer Ausgaben. Die verbleibenden Einnahmen gehen zu 25 Prozent an den Stool, 20 Prozent erhält die traditionelle Autorität in ihrer treuhänderischen Funktion und 55 Prozent die staatliche District Assembly für infrastrukturelle Verbesserungen in der Gegend (ebd.: Art. 267, Abs. 6). Der Stool bezeichnet in diesem Zusammenhang alle Mitglieder der Gemeinschaft ${ }^{1}$.

\section{Rechtspluralismus als Fundament gegenwärtiger Landrechte}

Gegenwärtige Landrechte in Ghana leiten sich aus der National Land Policy von 1999 ab (Ministry of Lands and Forestry 1999). Ziel dieser ersten umfassenden Bodenpolitik Ghanas war es, zentrale Herausforderungen in der Verwaltung von Land herauszuarbeiten und ein kohärentes Landrechtssystem zu entwickeln. Insbesondere hebt die National Land Policy hervor, dass es in der Vergangenheit an Maßnahmen mangelte, über welche die Vergabe von Land erfasst werden könne, um beispielsweise eine Mehrfachvergabe $\mathrm{zu}$ verhindern. Die wichtigste Herausforderung in Bezug auf gewohnheitsrechtlich verwaltetes Land seien unklare Grenzen und ein Mangel an detaillierten Landkarten. Darüber hinaus zählten großflächige Zwangsaneignungen vermeintlich unbewirtschafteter Flächen durch die Regierung, ein schwaches Landverwaltungssystem und konkurrierende Landnutzungsformen $\mathrm{zu}$ den größten Schwierigkeiten (Ministry of Lands and Forestry 1999).

An Gesetzgebungen mangelte es im Landsektor jedoch nicht. Insgesamt identifiziert die National Land Policy 166 unterschiedliche Gesetzgebungen mit Bezug zur Landverwaltung. Aufgrund von Überschneidungen, Widersprüchen und Schwachstellen bildeten diese jedoch kein kohärentes Landrechtssystem (Quaye 2006). Das sollte die National Land Policy ändern, indem sie die »sinnvolle Nutzung des nationalen Lands und all seiner natürlichen Ressourcen von allen Bevölkerungsschichten, die unter Berücksichtigung der Prinzipien des nachhaltigen Ressourcenmanagements unterschiedlichen sozio22.3.2016. 
ökonomischer Aktivitäten nachgehen und dabei funktionsfähige Ökosysteme erhalten, sicherstellt« (Ministry of Lands and Forestry 1999: 6, Übers. d. Autorin).

Um die National Land Policy umzusetzen, startete die Regierung 2003, finanziert durch die Weltbank und andere Geberorganisationen, das Land Adminstration Project (LAP) (World Bank 2003). Das Projekt bestand aus zwei Phasen. Die erste dauerte von 2003 bis 2011 und die zweite zunächst von 2011 bis 2016, wurde dann aber bis Februar 2018 verlängert. Das übergeordnete Ziel des LAP war die Sicherung von Landrechten, insbesondere mittels Registrierung und Formalisierung zuvor informeller Rechte. Entsprechend der neoliberalen Ausrichtung der Maßnahmen, liegt diesem Ziel die Annahme zugrunde, die Sicherung von Landrechten sei eine unabdingbare Voraussetzung von Wachstum, wirtschaftlicher Entwicklung und Armutsminderung sowie einer Zunahme landbezogener Investitionen und einer effizienteren Landnutzung (World Bank 2003). Mit der Sicherung von Landrechten waren institutionelle Reformen verbunden, die dazu dienten, das Landverwaltungssystem zu dezentralisieren. Fortan sollten unabhängig agierende Behörden unter dem Dach der neuen Lands Commission existieren und zu einer besseren Landverwaltung beitragen.

Neben der Harmonisierung von staatlichen und traditionellen Landrechten strebte das LAP insbesondere die Registrierung traditioneller Landrechtsansprüche sowie die Aufzeichnung der Grenzen traditionell verwalteten Lands an. Vor allem in ländlichen Gegenden sind traditionelle Landrechtsansprüche in der Regel nicht dokumentiert (Elhadary/ObengOdoom 2012: 63; Schoneveld/German 2014), was im Rahmen des LAP als ein Hindernis in der erfolgreichen Verwaltung von Land galt. Zur Stärkung der traditionellen Landverwaltung sollte, wie in der National Land Policy formuliert, das LAP lokale Customary Land Secretariats (CLS) unter dem Vorsitz traditioneller Autoritäten aufbauen. Diese sollen zu einer größeren Transparenz in der Verwaltung von gewohnheitsrechtlich genutztem Land beitragen. Aufgabe der CLS ist die Registrierung individueller Landrechtsansprüche sowie die Bearbeitung landbezogener Konflikte. Damit sollen sie eine Schnittstelle zwischen staatlicher und traditioneller Landverwaltung bilden (Schoneveld et al. 2011; Stacey 2015). In der Praxis hat sich jedoch herausgestellt, dass die CLS dazu beitragen, den Einfluss traditioneller Autoritäten in der Verwaltung und Vergabe von Land zu erhöhen. Denn den Vorsitz der CLS haben immer traditionelle Autoritäten inne. Fraglich ist daher, ob sie tatsächlich eine Neuerung darstellen oder nur eine durch den 
Staat geschaffene traditionelle Institution, die den Einfluss traditioneller Autoritäten in Landfragen stärkt. Nach Abschluss des Land Administration Project wurde deutlich, dass im Rahmen seiner Implementierung versäumt wurde zu hinterfragen, inwiefern chiefs in ihrem Handeln kontrolliert werden müssten, um sie daran zu hindern, sich Pachteinnahmen anzueignen, die ansonsten dem Gemeinwohl zustehen würden. Diese Problematik geht auf den Versuch zurück, in einem Zuge die staatliche Landverwaltung und die Verwaltung durch traditionelle Autoritäten zu stärken (World Bank 2013: ix).

Das gegenwärtige Landrecht unterscheidet drei Kategorien von Land: (1) state land, (2) customary land und (3) private land (Kasanga/Kotey 2001). Innerhalb dieser drei Kategorien differenziert es zwischen (1) state land und vested land, (2) stool, skin und family land sowie (3) individual und private land. State land sind Flächen, die sich der Staat im Laufe der Zeit im öffentlichen Interesse und für öffentliche Zwecke wie den Bau von Infrastruktur angeeignet hat. Die gesetzliche Grundlage dafür bildet der State Lands Act von 1962 (Republic of Ghana 1962b). Mit der Aneignung des Lands durch den Staat und nach einer gesetzlich festgelegten Entschädigungszahlung an die ehemaligen Landnutzer*innen erlöschen alle vorherigen Nutzungsrechte (Kasanga/Kotey 2001: 1). Vested land sind Flächen, die einer dualen Eigentumsstruktur unterliegen. Sie werden über den Administration of Lands Act reguliert (Republic of Ghana 1962a). Der Staat erhebt einen rechtlichen Anspruch auf das Land, überlässt die gewohnheitsrechtliche Verwaltung jedoch traditionellen Autoritäten. Der Zugang zu Land und die Nutzungsrechte verbleiben in diesem Zusammenhang in der landbesitzenden Gruppe, während der Staat die Einnahmen aus dem Land treuhänderisch im Sinne der jeweiligen Gemeinschaft verwaltet. Die durch das Land erzielten Einnahmen verteilt die staatliche Lands Commission an die lokale Regierung, traditionelle Autoritäten und Landbesitzer*innen, wobei sie einen Teil zur Deckung der administrativen Kosten einbehält (Amanor 2009: 97f.). Etwa 80 Prozent aller landwirtschaftlichen Flächen in Ghana fallen unter die Kategorie customary land, das traditionelle Autoritäten in traditionellen Landverwaltungssystemen auf der Grundlage von Gewohnheitsrecht verwalten (Kasanga/Kotey 2001: 1).

\section{Traditionelle Landverwaltungssysteme in Ghana}

Im Gegensatz zu formal-staatlichem Recht haben traditionelle Landverwaltungssysteme ihren Ursprung nicht in der staatlichen Politik, sondern beruhen auf verbal übermitteltem Gewohnheitsrecht, das gesellschaftliche Regeln 
und Normen widerspiegelt. Diese Regeln und Normen sind aus der jeweiligen Gemeinschaft heraus entstanden und historisch gewachsen. Sie sind das Ergebnis gesellschaftlicher Aushandlungsprozesse und variieren von Region zu Region und von Dorf zu Dorf. Die Ausgestaltung traditioneller Landverwaltungssysteme unterscheidet sich daher zwischen den Regionen Ghanas. Unterschiede finden sich nicht zuletzt auch in den Bezeichnungen für gewohnheitsrechtlich verwaltetes Land, etwa stool land, skin land, clan oder family land. Die Begriffe leiten sich aus den unterschiedlichen Bräuchen und Herrschaftsinstitutionen ab. Im Süden Ghanas sitzen traditionelle Autoritäten bei ihrer Ernennung zum chief sowie zu offiziellen Anlässen auf hölzernen Schemeln (stools), im Norden auf Tierhäuten (skins), welche die Macht der chiefs symbolisieren. Die Zeremonie der Ernennung eines chiefs wird daher auch als enstoolment oder enskinment bezeichnet ${ }^{2}$. Die Begriffe stool land und skin land gehen auf diesen Brauch zurück. Stool oder skin bezeichnet darüber hinaus die Gesamtheit einer traditionellen Gemeinschaft. Diese umfasst die traditionellen Autoritäten und ihre »Untergebenen« (subjects), die über die Anerkennung traditioneller Autoritäten maßgeblich zu deren Legitimation beitragen ${ }^{3}$.

Gewohnheitsrechtliche Ansprüche auf Land haben in der Regel eine lange Geschichte. In vielen Fällen werden sie über die erste Niederlassung einer Gruppe in einer bestimmten Gegend legitimiert (Berry 2009b). Kennzeichnend für gewohnheitsrechtlich verwaltetes Land ist jedoch die Koexistenz vielfacher, parallel existierender Rechte. Unterschiedliche Personen tragen, abhängig von ihrem Status und ihrer Position innerhalb der Bezugsgruppe, die Verantwortung für die Vergabe dieser Rechte (Berry 1993). Die Nutzungsrechte für gewohnheitsrechtlich verwaltetes Land liegen in der Gemeinschaft, die erfolgreich ihren Anspruch auf das Land geltend macht. Diese Gemeinschaft hat den Allodial Title inne, den höchsten Besitzanspruch im Gewohnheitsrecht, über dem kein übergeordnetes Interesse existiert (Kasanga/Kotey 2001: 13). Bei stool und skin lands repräsentiert der zuständige chief den Allodial Title (Republic of Ghana 1992: Art. 268, Abs. 261) und verwaltet als Treuhänder für seine Gemeinschaft das Land.

In Bezug auf die Verwaltung von Land überträgt er Verantwortung an unter ihm stehende sub-chiefs oder village chiefs (Odikros), die für die Vergabe von

2 Vorsitzender des National Office of the Administrator of Stool Lands, Interview am 22.3.2016.

3 Vorsitzender des National Office of the Administrator of Stool Lands, Interview am 22.3.2016. 
Abbildung 4: Darstellung des durch einen paramount chief verwalteten Lands

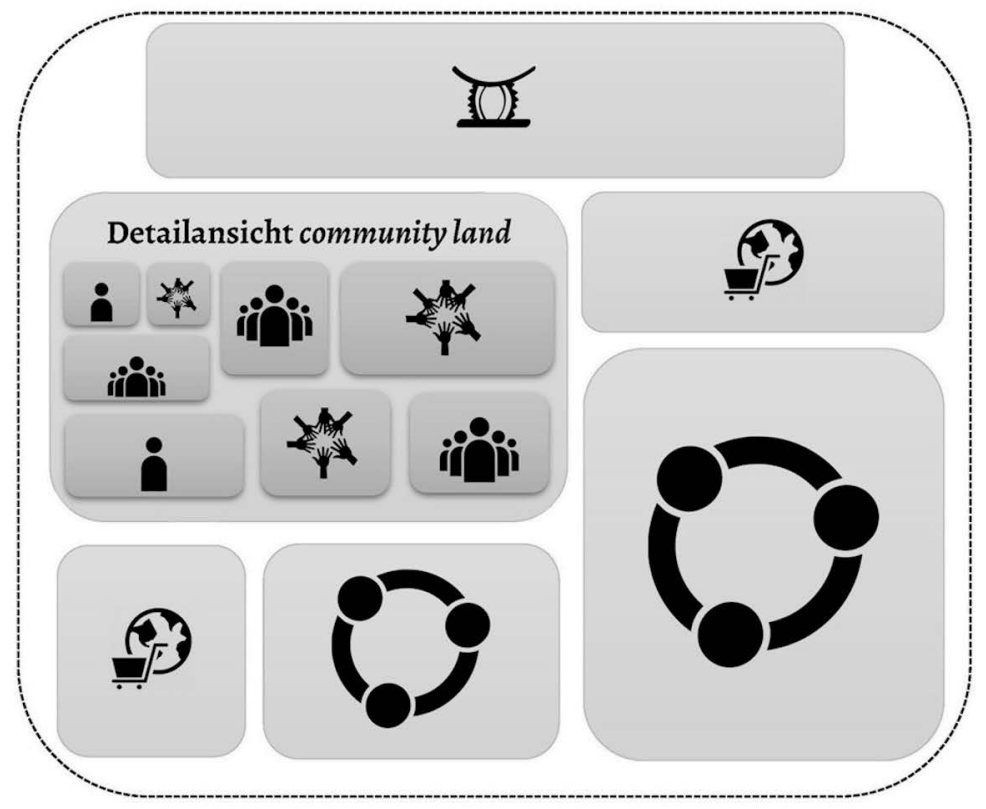

\section{Legende}

Stool land des paramount oder
sub-chiefs

Quelle: Eigene Darstellung

Landnutzungsrechten auf Dorfebene zuständig sind. Alle chiefs können - unabhängig von ihrer hierarchischen Stellung - einen Teil des durch sie verwalteten Lands für sich selbst beanspruchen. In Abgrenzung zu gemeinschaftlich genutztem Land (communal land) wird dieses wiederum als stool oder skin land bezeichnet. Über dieses Land können die chiefs allein verfügen, ohne mit den 
Mitgliedern der Gemeinschaft Rücksprache zu halten. Bei family land oder clan land verfügt das zuständige (erweiterte) Familienoberhaupt über den Allodial Title und damit über das Recht Land zu vergeben. Landnutzungsrechte von Individuen oder Familien, die Teil der landbesitzenden Gruppe sind, beruhen entweder auf dem customary freehold oder dem usufruct. Der customary freehold ist ein dauerhaftes Recht und ermöglicht es neben der eigenen Nutzung, Land an Nachkommen zu vererben und anderen Landnutzer*innen (z.B. im Rahmen von sharecropping-Vereinbarungen oder Pachtverträgen) zur Verfügung zu stellen.

Da die Gemeinschaft immer die Lebenden, Toten und Ungeborenen umfasst, verbietet das Gewohnheitsrecht den Verkauf von Land. Der Verkauf von Land würde bedeuten, die eigene Entscheidung über diejenige der Toten und Ungeborenen zu stellen (Ahmed et al. 2018: 575). Der usufruct ist für Mitglieder der Gemeinschaft ein permanentes Recht. Im Unterschied zum customary freehold erlaubt der usufruct es ihnen jedoch nicht, das Land an Dritte weiterzugeben und ist an die Nutzung des Lands geknüpft. Migrant*innen - Landnutzer*innen, die ursprünglich aus anderen Gegenden oder Ländern kommen - erhalten meist temporäre Nutzungsrechte. Diese sind zudem mit einer geldlichen Gegenleistung oder einer Naturalpacht (sharecropping) verbunden. Jährlich oder nach jeder Ernte sind sie verpflichtet einen Teil der Ernteerzeugnisse an den sub-chief bzw. die Landbesitzer*innen, die das Land zur Verfügung stellen, abzugeben. Gängige sharecropping-Vereinbarungen in Ghana sind abusa und abunu. Abusa bedeutet, dass zwei Drittel der erwirtschafteten Erzeugnisse an den Pächter oder die Pächterin gehen und die Landbesitzer*innen das verbleibende Drittel bekommen. Dafür sind die Pächter*innen selbst für die Vorbereitung des Lands zuständig. Abunu bedeutet, dass Pächter*innen und Landbesitzer*innen die Ernteerzeugnisse gleichmäßig teilen. Unter dieser Vereinbarung tragen Landbesitzer*innen meist gemeinsam mit den Pächter*innen die Verantwortung für die Verwaltung der Farm.

Investoren erhalten Nutzungsrechte an traditionell verwaltetem Land ebenfalls über Pachtvereinbarungen. Verfassungsrechtlich ist die maximale Pachtdauer von Land für Ausländer*innen auf 50 Jahre begrenzt (Republic of Ghana 1992: Art. 266, Abs. 4). Ghanaer*innen hingegen können Land auch für einen längeren Zeitraum pachten. Investoren, die traditionell verwaltetes Land pachten möchten, müssen - unabhängig davon von wem sie das Land pachten - den für die Landverwaltung zuständigen chief persönlich um sein Einverständnis bitten. Um traditionellen Autoritäten ein Anliegen unterbreiten zu können, ist es üblich ihnen zuvor als Zeichen des Respekts 
eine symbolische Gabe $\mathrm{zu}$ überreichen. Traditionell war dies eine Flasche Schnaps, im Norden Ghanas sind es manchmal auch Kolanüsse. Seit einigen Jahren werden Schnaps oder Kolanüsse immer häufiger durch kleine Summen Geld ersetzt, die - unter Rückbezug auf den früheren Brauch - als drink money bekannt sind. Mit dem zunehmenden Interesse von Investoren an der großflächigen Aneignung von Land begannen chiefs große Geldsummen $\mathrm{zu}$ verlangen, um Zugang $\mathrm{zu}$ Land $\mathrm{zu}$ ermöglichen (Blocher 2006; Schoneveld/German 2014). Die Bezeichnung drink money und der damit verbundene Rückbezug auf einen üblichen Brauch rechtfertigt diese Vorgehensweise, an die sich Investoren halten müssen, um erfolgreich mit ihrem Vorhaben zu sein.

Abbildung 5: Traditionelle Landrechte in Ghana

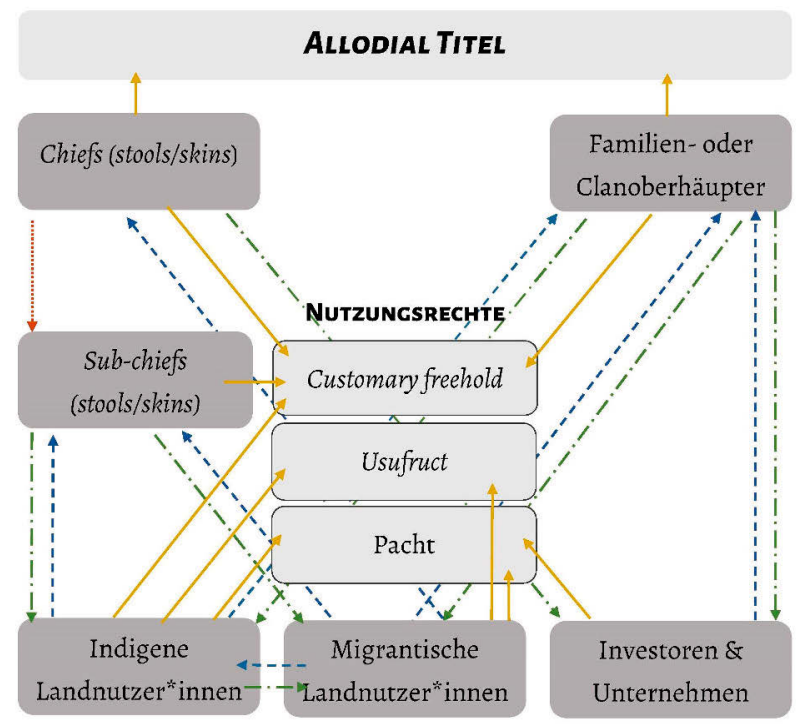

$\underline{\text { Legende }}$

$\ldots$ Übertragen Verantwortung

$\rightarrow$ Legitimieren Rechtsansprüche

$-\rightarrow$ Übertragen Rechte

$\longrightarrow$ Verfügen über

Quelle: Eigene Darstellung 


\subsection{Traditionelle Herrschaft in der Ashanti und Northern Region}

Wie in vielen anderen Ländern Afrikas ist traditionelle Herrschaft in Ghana einer kleinen Gruppe privilegierter Personen vorbehalten. Den Anspruch auf dieses Privileg machen sie geltend, indem sie angeben, patrilineale oder matrilineale Nachkommen einer bestimmten Abstammungslinie (lineage) zu sein. Diese trägt oftmals die Bezeichnung royal family. Dabei handelt es sich um die Abstammungslinie, dessen Vorfahren als Gründer*innen der jeweiligen Gemeinschaft gelten. Traditionelle Gesellschaften unterteilen sich in zwei Sozialgruppen: eine kleine Gruppe »Adeliger« (royals), welche die traditionelle Elite bildet und einen Großteil »Nichtadeliger « (commoners). Ausschließlich Personen aus der traditionellen Elite können den Posten einer traditionellen Autorität erlangen (Tonah 2012: 4).

Das chieftaincy-System in der Ashanti Region ist streng hierarchisch organisiert, mit dem Oberhaupt der Ashanti, dem Asantehene, an der Spitze. hene bedeutet auf Akan König oder chief (Agyekum 2006: 225). Asantehene bedeutet also König der Ashanti. Der Asantehene ist der Inhaber des Golden Stool und hat den Vorsitz des Ashanti Regional House of Chiefs sowie des Kumasi Traditional Council inne. Zwischen dem Tod eines Asantehene und der Wahl seines Nachfolgers vertritt ihn der Mamponghene, der Inhaber des Silver Stool und Vorsitzender des Mampong Traditional Council. Insgesamt umfasst das traditionelle Herrschaftssystem der Ashanti 36 traditional councils, die jeweils für eine bestimmte Gegend (traditional areas) zuständig sind. Einen traditional council bilden ein paramount chief (omanhene) und unter ihm stehende sub-chiefs, auch als elders bezeichnet, sowie queenmothers. Der paramount chief hat den Vorsitz des traditional council. Einige der sub-chiefs stehen den zahlreichen kleineren Gemeinschaften innerhalb einer traditional area vor und sind dem paramount chief zu Loyalität verpflichtet. Andere haben eine beratende Funktion.

In der Northern Region gibt es, anders als in der Ashanti Region, kein einheitliches chieftaincy-System und damit auch nicht nur ein einziges Oberhaupt wie den Asantehene. Zum Zeitpunkt der Feldforschung ${ }^{4}$ gab es in der Northern Region fünf traditional councils, an deren Spitze jeweils ein traditionelles Oberhaupt steht. Der untersuchte Fall befindet sich im Zuständigkeitsbereich 
des Dagomba Traditional Council, dem größten traditional council der Region. Der Dagomba Traditional Council hat seinen Sitz in Yendi und sein traditionelles Oberhaupt ist der Ya Naa (Ahorsu 2014: 100). Naa (oder Na) bedeutet König oder chief in Dagbani, der Sprache der Dagombas (Brukum 2005: 27; Ladouceur 1972: 98). Ya Naa bedeutet king of strength (Iddrisu 2013: 25). Ähnlich wie das Ashanti chieftaincy-System ist das traditionelle Herrschaftssystem der Dagombas hierarchisch organisiert. An der Spitze befindet sich der Ya Naa. Unter ihm stehen paramount und sub-chiefs. Jeder chief ist dem in der Hierarchie über ihm stehenden chief zu Loyalität verpflichtet (Tonah 2012: 6).

\section{Ernennung traditioneller Autoritäten}

Eine Gemeinsamkeit traditioneller Autoritäten in allen Regionen - egal ob paramount chiefs, sub-chiefs oder andere Posten innerhalb der traditionellen Elite - ist, dass sie ernannt und nicht gewählt werden. Die Ernennung ist so genannten kingmakers oder queenmakers vorbehalten, die ebenfalls aus einer royal family abstammen müssen, selbst jedoch niemals Anwärter*innen auf den entsprechenden Posten sein können. Vor der Ernennung beraten sich diese mit anderen elders aus der traditionellen Elite, um eine* $n$ geeignete* $n$ Kandidat*in auszuwählen.

Das chieftaincy-System in der Ashanti Region ist matrilineal, was so viel bedeutet wie »in der Erbfolge der mütterlichen Linie folgend« (Dudenredaktion o.J.). Matrilineal ist nicht mit matriarchal zu verwechseln. Anders als in einem Matriarchat sind hohe Posten in matrilinealen Gemeinschaften nicht zwangsläufig Frauen vorbehalten. Das gilt auch für das chieftaincy-System in der Ashanti Region, in der die chief-Posten, bis auf wenige Ausnahmen, ausschließlich von Männern besetzt werden. Weibliche chiefs können nur ernannt werden, wenn es keinen männlichen Anwärter für den Thron gibt (BoafoArthur 2003: 135). Die Matrilinearität des chieftaincy-Systems in der Ashanti Region verkörpert die Königinmutter (queenmother). Geeignete Kandidaten für den chief-Posten müssen aus ihrer Blutlinie abstammen und sie ist als kingmaker für ihre Nominierung zuständig. Dementsprechend kann sie ihren eigenen Sohn nominieren, wie es auf den aktuellen Asantehene Osei Tutu II zutrifft. Sie kann aber auch den Sohn ihrer Schwester, den Bruder ihrer Mutter oder den Sohn ihrer Tante mütterlicherseits nominieren. Ihre Nominierung muss durch die elders des traditional council abgesegnet werden. Nur aufgrund berechtigter Einwände dürfen diese die Nominierung ablehnen. Lehnen die elders drei Nominierte in Folge ab, dürfen sie selbst einen 
Kandidaten nominieren und ernennen. Diese Prozedur der Ernennung gilt für alle unterschiedlichen chief-Posten in der Ashanti-Region ${ }^{5}$.

Das chieftaincy-System der Dagombas ist patriarchal. Die Ernennung traditioneller Autoritäten berücksichtigt also die väterliche Linie der Erbfolge und erfolgt, wie auch in Ashanti, über die kingmaker. Der Großteil aller chiefs in Dagbon sind Männer. In drei Städten (Kukulogu, Kpatuya, Gundogu) sind jedoch Frauen die chiefs. Diese drei skins sind den Töchtern des Ya Naa vorbehalten (Boafo-Arthur 2003: 135f.). Anders als im chieftaincy-System der Ashanti unterscheidet sich die Ernennung des Ya Naa von derjenigen der unter ihm stehenden traditionellen Autoritäten. Paramount und sub-chiefs müssen, wie auch in Ashanti, jeweils aus einer bestimmten Abstammungslinie kommen, die einem bestimmten skin zugeordnet ist ${ }^{6}$. Einige dieser skins sind so genannte gate skins, die Zugang zu einem höheren skin, also einer höheren Position im chieftaincy-System ermöglichen (Ladouceur 1972: 98). Andere skins bieten diese Möglichkeit nicht. Ihren Inhaber*innen bleibt es verwehrt, in der Hierarchie aufzusteigen. Neben der zentralen Rolle der kingmaker in der Ernennung neuer chiefs, bedarf diese immer auch der Zustimmung des amtierenden Ya Naa sowie höher stehender chiefs (MacGaffey 2006: 89). Die Ernennung zum chief ist damit maßgeblich an gute Beziehungen zur traditionellen Elite geknüpft.

Die Möglichkeit zum Ya Naa ernannt zu werden, besteht ausschließlich für die paramount chiefs der drei gate skins Karaga, Mion und Savelugu. Diese skins sind den Söhnen des $\mathrm{Ya}$ Naa vorbehalten, so dass nur der Sohn eines ehemaligen Ya Naas selbst Ya Naa werden kann. Die Ernennung des Ya Naas erfolgt über ein rotierendes System, in dem sich die drei lineages der gate skins in der Thronfolge ablösen (Ahorsu 2014: 100f.). Eine traditionelle Kommission von vier kingmakers ernennt den neuen $\mathrm{Ya}$ Naa. Ihre Entscheidung beruht auf der Qualifikation des zukünftigen Ya Naas sowie auf Wahrsagen durch ein Orakel (MacGaffey 2006: 88).

\section{Traditionelle Landverwaltung in den Forschungsregionen}

Laut Verfassung obliegt die Verwaltung von Land in beiden Forschungsregionen den jeweiligen stools und skins, die treuhänderisch, im Einklang mit tra-

6 Research Officer des Regional House of Chiefs der Northern Region, Interview am 8.3.2017. 
ditionellen Rechten und im Sinne der Mitglieder der Gemeinschaft darüber verfügen können (Republic of Ghana 1992: \$267, Abs. 1). Die traditionellen Rechte in Bezug auf die Verwaltung von Land in den beiden Forschungsregionen weisen viele Ähnlichkeiten, aber auch einige Unterschiede auf.

Das Land in der Agogo Traditional Area lässt sich in stool land, communal land, family land und individual land unterteilen. Stool land ist immer einem bestimmten stool zugeordnet und steht dem Inhaber des jeweiligen stools also dem paramount chief oder dem sub-chief - zur freien Verfügung. Communal land ist Land, dass der gesamten Gemeinschaft - zu der beispielsweise auch Migrant*innen gehören - zur Verfügung steht. Je nach Bedarf vergibt der sub-chief temporäre Nutzungsrechte für dieses Land. Für Mitglieder der Gemeinschaft sind diese Nutzungsrechte permanent, so lange sie das Land bestellen. Auf family land können diejenigen Familien Anspruch erheben, die glaubhaft machen können, dass ihre Vorfahren die ersten Nutzer*innen des Lands waren. Rechte auf dieses Land sind in der Regel permanent und vererbbar, auch wenn das Land eine Zeitlang brach liegt. Individual lands bezeichnet Flächen, für die Landnutzer*innen verbriefte traditionelle Rechte, also Rechte die durch eine traditionelle Autorität schriftlich bestätigt und nicht nur mündlich festgehalten wurden, erworben haben. Solche Rechte kann nur der paramount chief gewähren und sie sind in der Regel mit einer Gegenleistung, z.B. einer Beteiligung an der Ernte verbunden ${ }^{7}$.

Für die alltägliche Vergabe und Verwaltung von Land auf Dorfebene ist der sub-chief zuständig. Eine Ausnahme bildet family land, das der jeweilige family head, das Oberhaupt der Familie, verwaltet. Für die Vergabe von Land für kommerzielle Zwecke ist auf Dorfebene ebenfalls der sub-chief zuständig, wobei er den paramount chief darüber in Kenntnis setzen und die Einnahmen mit ihm teilen muss. Für Flächen, die über die Dorfebene hinausgehen, ist der paramount chief zuständig ${ }^{8}$. Seine Aufgabe ist es in diesem Fall allerdings, die jeweiligen sub-chiefs darüber zu informieren und sie zu konsultieren. Die Aufgabe der sub-chiefs ist es wiederum, die Informationen an ihre Gemeinschaft

7 Registerführer des Agogo Traditional Council und Leiter des Agogo Customary Land Secretariat, Interview am 20.4.2016; Landnutzer Nsonyameye und Vorsitzender der Agogoman mma kuo, Interview am 16.3.2017.

8 Registerführer des Agogo Traditional Council und Leiter des Agogo Customary Land Secretariat, Interview am 20.4.2016. 
weiterzuleiten und die Meinung der jeweiligen Landnutzer*innen einzuholen ${ }^{9}$.

In Dagbon verfügt, laut traditionellem Recht, der Ya Naa über jegliches skin land (Ahorsu 2014: 101). Er überträgt die Aufgabe der Landverwaltung jedoch an unter ihm stehende paramount chiefs. In der Projektgegend sind das die chiefs von Sang (Sang Lana), Mion (Mion Lana), Salankpang (Salankpang Lana), Zakpalsi (Zakpalsi Lana) und Tijo (Tijo Naa). Die alltägliche Verwaltung von Land für kleinbäuerliche Landwirtschaft übernehmen sub-chiefs, die einzelnen Dörfern vorstehen. Auf Dorfebene können Familienoberhäupter zur Bestreitung ihres Lebensunterhalts für ihre Familie ein Stück Land frei auswählen und müssen nur den sub-chief darüber informieren. Migrant*innen erhalten Zugang zu Land durch den sub-chief, dem sie als Zeichen des Respekts einige Kolanüsse mitbringen. Einmal zugestandene Landnutzungsansprüche können in der Regel weder für Mitglieder der Gemeinschaft noch für Migrant*innen rückgängig gemacht werden. Der Ya Naa oder der paramount chief können jedoch Land im Interesse der Gemeinschaft vergeben. Indigenen, die in diesem Zuge Land verlieren, erhalten einen entsprechenden Ersatz. Migrant"innen haben keinen Anspruch auf Entschädigung (Tsikata/Yaro 2011: 18). Die Vergabe von Land für die kommerzielle Nutzung obliegt den paramount chiefs, die ebenfalls dafür zuständig sind, alle Familienoberhäupter ihrer Gemeinschaft sowie die unter ihnen stehenden sub-chiefs zu informieren. Darüber hinaus muss die Landvergabe durch den $\mathrm{Ya} N a a$ abgesegnet werden. Dabei handelt es sich um einen formellen Akt. Er segnet die entsprechende Landvergabe mit seiner Unterschrift $a b$ und erhält im Gegenzug einen Anteil des Geldes.

Die Verwaltung und Vergabe von Land in den beiden Forschungsregionen weist zum größten Teil Gemeinsamkeiten auf. Ein Unterschied betrifft die Vergabe von Land für kommerzielle Zwecke. In der Ashanti Region kontrollieren die paramount chiefs die Vergabe von Land für kommerzielle Zwecke, indem sie es selbst vergeben oder die Vergabe von durch sub-chiefs verwaltetem Land absegnen und in diesem Zuge Anspruch auf einen Teil der Einnahmen erheben. Paramount chiefs in der Ashanti Region - mit Ausnahme der Kumasi Traditional Area - sind niemandem in der Hierarchie höher stehenden zu Rechenschaft verpflichtet. Paramount chiefs in der Northern Region hingegen sind dem Ya Naa zu Rechenschaft verpflichtet, der die Vergabe von Land am 20.4.2016. 
134 Umkämpfter Zugang zu Land

für kommerzielle Zwecke in letzter Instanz absegnet. Diese Absegnung ist in der Regel eine Formsache und schränkt die paramount chiefs nicht in ihrer Entscheidungsfreiheit ein. 